\title{
Synthesis and Biological Evaluation of a Novel Series of Pyrazolylcoumarins as Anti-Inflammatory and Antioxidant Agents
}

\section{Synthesis of 3-(1H-pyrazol-5-yl)-2H-chromen-2-one derivatives}

\author{
Authors \\ P. Aragade, R. Venkatnarayanan, P. Patil \\ Affiliation \\ RVS College of Pharmaceutical Science, Sulur, Coimbatore, Tamilnadu, India
}

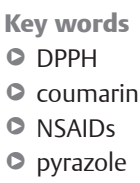

received 10.07 .2012

accepted 13.11.2012

\section{Bibliography}

DOI http://dx.doi.org/

10.1055/s-0032-1331225

Published online:

January 8, 2013

Drug Res 2013;

63: 28-33

(c) Georg Thieme Verlag KG

Stuttgart · New York

ISSN 2194-9379

\section{Correspondence}

Prashant. D. Aragade, MPharm RVS College of Pharmaceutical Science

242 B

Trichy Road, Sulur

Coimbatore- 641402

Tamilnadu

India

Tel.: + 91/422/268 7604

Fax: $+91 / 422 / 2687604$

prashant.argade@gmail.com

\section{Abstract \\ $\nabla$}

A new series of pyrazolylcoumarins (4a-k) was synthesized by reaction of appropriate dibromochalcones (3a-k) with phenyl hydrazine in ethanol. Structures of all new synthesized compounds were characterized on the basis of elemental analysis and spectral data (UV, IR and ${ }^{1} \mathrm{H}$ NMR). The title compounds were screened for in vivo anti-inflammatory activities at a dose of $200 \mathrm{mg} /$ $\mathrm{kg}$ b.w. Among the 11 prepared compounds, Compounds $\mathbf{4 c}, \mathbf{d}, \mathbf{h}$ and $\mathbf{i}$ exhibited significant

\section{Introduction}

Nonsteroidal anti-inflammatory drugs (NSAIDs) have been recognized as important class of therapeutic agents for the alleviation of pain and inflammation associated with a number of pathological conditions. However, long term use of NSAIDs has been associated with several side effects such as gastrointestinal mucosal damage, bleeding, intolerance and renal toxicity [1-4]. Thus, there is an urgent need for new targets that are required for the design and development of novel anti-inflammatory agents as an alternative to NSAIDs. Extensive research has been directed towards improving their pharmacological profile that led to the discovery of multiple isoforms of cyclooxygenase (COX) that are differently regulated $[5,6]$. The discovery of the inducible isoform of cyclooxygenase enzyme (COX-2) spurred the search for anti-inflammatory agents devoid of the undesirable effects associated with classical NSAIDs. Recently, a novel class of selective COX-2 inhibitors has been discovered. Among this class, celecoxib was shown to be a potent and gastrointestinal (GI) safe anti-inflammatory analgesic agent. It is considered a typical model of pyrazole containing, diaryl-heterocyclic template that is known to selectively inhibit COX-2 anti-inflammatory activity in model of acute inflammation such as carrageenan-induced rat edema paw while compound $\mathbf{4 d}$ showed considerable activity in model of chronic inflammation such as adjuvant-induced arthritis along with minimum ulcerogenic index and were compared with diclofenac $(13.5 \mathrm{mg} / \mathrm{kg}$ b.w.) as a standard drug. Additionally, the synthesized compounds were evaluated for their in vitro antioxidant activity. Compound $\mathbf{4 k}$ was found to be the most active antioxidant in the series compared with standard vitamin $\mathrm{C}$.

[7]. Furthermore, several other compounds containing pyrazole functionality were also reported to exhibit anti-inflammatory activity [8-12].

Reactive oxygen species (ROS) are implicated in the induction and prolongation of inflammatory process [13]. Interestingly a number of therapeutically useful NSAID's have been shown to act by virtue of their free radical scavenging activity[14-16]. Antioxidants are the compounds that prevent oxidative damage induced by free radicals and ROS. Thus, antioxidant therapy has also gained immense importance in the treatment of the above mentioned diseases [17].

Coumarin derivatives are an interesting class of heterocyclic systems, since coumarin ring consist an essential core moiety for a variety of natural and synthetic biologically active compounds [18-21]. The biological activities include anticoagulation, antibiotic, antifungal, antipsoriasis, antitumor, anti-HIV, anti-inflammatory, etc. Moreover, since the combination of 2 pharmacophores on the same scaffold is a well established approach to the synthesis of more potent drugs, we decided to incorporate the pyrazole ring moiety in the coumarin derivatives and to examine the influence of this modification on their activities as anti-inflammatory and free radical scavengers. 


\section{Experimental}

All research chemicals were purchased from Sigma-Aldrich (St. Louis, Missouri, USA) or Lancaster Co. (Ward Hill, MA, USA) and used as such for the reactions. Solvents except laboratory reagent grade were dried and purified according to the literature when necessary.

Reactions were monitored by thin-layer chromatography (TLC) on pre-coated silica gel plates from E. Merck and Co. (Darmstadt, Germany). Melting points of synthesized compounds were determined in Thermonik (Mumbai, India) melting point apparatus and are uncorrected, UV spectra were recorded on Thermospectronic (Rochester, NY, USA) and IR spectra were recorded on Thermo Nicolet IR200 FT-IR Spectrometer (Madison, WI, USA) by using $\mathrm{KBr}$ pellets. The ${ }^{1} \mathrm{HNMR}$ were recorded on Bruker AVANCE 300 (Bruker, Rheinstetten/Karlsruhe, Germany) using DMSO- $\mathrm{d}_{6}$ as solvent. Chemical shifts are reported in $\delta$ ppm units with respect to TMS as internal standard. The purity of compounds was examined by TLC on silica gel plate using chloroform and methanol (10:1) as mobile phase and iodine vapours as visualizing agent. The anti-inflammatory activity was carried out using digital plethysmometer (Ugo-Basile, Italy). A series of 3-[3-(substituted phenyl)-1-phenyl-1H-pyrazol-5-yl]-2H-chromen-2-one (4a-k) was synthesized by earlier reported method [23]. The physico-chemical and spectral data of synthesized compounds 4a-k is summarized in $\bullet$ Table 1, 2 respectively.

\section{Pharmacological Evaluation}

$\nabla$

Albino mice of either sex weighing 20-25g were used for acute toxicity studies and analgesic activity. Healthy male albino adult rats weighing $150-230 \mathrm{~g}$ were used for various pharmacological screenings. Animals were procured from Department of Pharmacology, RVS College of Pharmaceutical Sciences, Sulur, Tamilnadu, India, (1012/C/06/CPCSEA) and housed individually in polypropylene cages, maintained under standard conditions of alternating $12 \mathrm{~h}$ light and dark cycles at a constant temperature (25 $\pm 2^{\circ} \mathrm{C}$ and $35-60 \%$ relative humidity). Animals were fed with standard rat pellet diet, (Hindustan Lever Ltd., Mumbai, India) and water ad libitum.

\section{Acute toxicity [24]}

The acute toxicity test was carried out according to the Organization for Economic Co-operation and Development (OECD) guidelines to establish the effective dose of test compounds after obtaining ethical clearance from Animal Ethics Committee of RVS College of Pharmaceutical Sciences, Sulur (India). Albino mice of either sex weighing between 20 and $25 \mathrm{~g}$ were grouped into 12 groups of 6 animals each, starved for $24 \mathrm{~h}$ with water ad libitum prior to test. On the day of the experiment animals were administered with different compounds to different groups in an increasing dose of 10,20,100,200, 1000 and $2000 \mathrm{mg} / \mathrm{kg}$ body weight orally. The animals were then observed continuously for $3 \mathrm{~h}$ for general behavioral, neurological, autonomic profiles and then every $30 \mathrm{~min}$ for next $3 \mathrm{~h}$ and finally for next $24 \mathrm{~h}$ or till death.

\section{Acute anti-inflammatory activity}

In vivo acute anti-inflammatory activity was evaluated using carrageenan-induced rat paw edema assay model of inflammation by adopting the method of Winter et al. [25] for the compounds listed in Table 3. Male albino rats (170-220g) were fasted with free access to water at least $12 \mathrm{~h}$ prior to experiments and were divided randomly into 13 groups of 6 each. Control group received $1 \mathrm{ml}$ of $0.5 \%$ sodium carboxymethyl cellulose (sodium CMC), standard group received $13.5 \mathrm{mg} / \mathrm{kg}$ of diclofenac and test groups received $200 \mathrm{mg} / \mathrm{kg}$ of synthesized compounds (4a-k). The rats were dosed orally, $1 \mathrm{~h}$ later; a subplantar injection of $0.05 \mathrm{ml}$ of $1 \%$ solution of carrageenan in sterile distilled water was administered to the left hind footpad of each animal. The paw edema volume was measured with a digital plethysmometer at $0,1,2,3,4,5 \mathrm{~h}$ after carrageenan injection. Paw edema volume was compared with vehicle control group and percent reduction was calculated as 1-(edema volume in the drug treated group/edema volume in the control group $) \times 100$.

\section{Chronic anti-inflammatory activity}

In vivo chronic anti-inflammatory activity was assessed using adjuvant-induced arthritis assay model of inflammation by utilizing the earlier reported method of Newbould [26]. Normally fed male albino rats weighing between 150 and $230 \mathrm{~g}$ were used for the experiment. On day 1, $0.1 \mathrm{ml}$ of heat-killed Mycobacterium tuberculosis (Freund's adjuvant complete) was injected into the left hind footpad of each rat. The rats were kept in cages for 15 days. On day 15, all animals with 'developed' arthritis were used for the study and were divided into 6 groups of 6 rats each for various treatments as shown in $\bullet$ Table 4. Control group received $1 \mathrm{ml}$ of $0.5 \%$ sodium CMC, standard group received $13.5 \mathrm{mg} / \mathrm{kg}$ b.w. of diclofenac. Test groups received $200 \mathrm{mg} / \mathrm{kg}$ b.w. of selected compounds $\mathbf{4 c}, \mathbf{d}, \mathbf{h}$ and $\mathbf{i}$. All compounds were administered orally from day 16 to 19 . The hind paw volumes,

Table 1 Physico-chemical data of 3-[3-(substituted phenyl)-1-phenyl-1H-pyrazol-5-yl]-2H-chromen-2-one derivatives (4a-k).

\begin{tabular}{|c|c|c|c|c|c|c|}
\hline Compound & $\mathbf{R}^{\mathbf{a}}$ & Yield (\%) & M. P. $\left({ }^{0} \mathrm{C}\right)$ & $\mathrm{Rf}^{\mathrm{b}}$ & Formula & M. W. \\
\hline $4 a$ & $\mathrm{H}-$ & 53 & $158-260$ & 0.38 & $\mathrm{C}_{24} \mathrm{H}_{16} \mathrm{~N}_{2} \mathrm{O}_{2}$ & 364 \\
\hline $4 b$ & 4-OMe- & 83 & $120-122$ & 0.27 & $\mathrm{C}_{25} \mathrm{H}_{18} \mathrm{~N}_{2} \mathrm{O}_{3}$ & 394 \\
\hline $4 c$ & $4-\mathrm{Cl}-$ & 76 & $98-100$ & 0.48 & $\mathrm{C}_{24} \mathrm{H}_{15} \mathrm{ClN}_{2} \mathrm{O}_{2}$ & 398 \\
\hline $4 d$ & $2,4-(\mathrm{Cl})_{2^{-}}$ & 92 & $126-130$ & 0.24 & $\mathrm{C}_{24} \mathrm{H}_{14} \mathrm{Cl}_{2} \mathrm{~N}_{2} \mathrm{O}_{2}$ & 433 \\
\hline $4 \mathrm{e}$ & $4-\mathrm{NMe}_{2}^{-}$ & 70 & $148-150$ & 0.46 & $\mathrm{C}_{26} \mathrm{H}_{21} \mathrm{~N}_{3} \mathrm{O}_{2}$ & 407 \\
\hline $4 \mathrm{f}$ & $3-\mathrm{NO}_{2}^{-}$ & 67 & $100-102$ & 0.25 & $\mathrm{C}_{24} \mathrm{H}_{15} \mathrm{~N}_{3} \mathrm{O}_{4}$ & 409 \\
\hline $4 \mathrm{~g}$ & 4-Me- & 74 & $90-92$ & 0.36 & $\mathrm{C}_{24} \mathrm{H}_{18} \mathrm{~N}_{2} \mathrm{O}_{2}$ & 378 \\
\hline $4 \mathrm{~h}$ & 3-OMe- & 83 & $136-138$ & 0.32 & $\mathrm{C}_{25} \mathrm{H}_{18} \mathrm{~N}_{2} \mathrm{O}_{3}$ & 394 \\
\hline $4 i$ & 4-F- & 64 & $190-192$ & 0.29 & $\mathrm{C}_{24} \mathrm{H}_{15} \mathrm{FN}_{2} \mathrm{O}_{2}$ & 382 \\
\hline $4 \mathrm{j}$ & $2-\mathrm{NO}_{2}^{-}$ & 71 & $208-210$ & 0.33 & $\mathrm{C}_{24} \mathrm{H}_{15} \mathrm{~N}_{3} \mathrm{O}_{4}$ & 409 \\
\hline $4 k$ & $4-\mathrm{OH}-$ & 76 & $182-184$ & 0.42 & $\mathrm{C}_{24} \mathrm{H}_{16} \mathrm{~N}_{2} \mathrm{O}_{3}$ & 380 \\
\hline
\end{tabular}

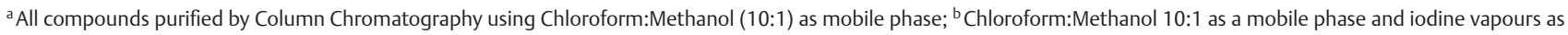
visualizing agent 
Table 2 Spectral Data of 3-[3-(substituted phenyl)-1-phenyl-1H-pyrazol-5-yl]-2H-chromen-2-one derivatives (4a-k).

\begin{tabular}{|c|c|c|c|c|c|}
\hline Compd & $\mathbf{R}$ & UV $\left(\mathrm{CH}_{3} \mathrm{OH}\right)$ & $\mathrm{IR}\left(\mathrm{KBr}, \mathrm{cm}^{-1}\right)$ & ${ }^{1} \mathrm{H}-\mathrm{NMR}$ (DMSO-d $\left.{ }_{6} \delta, \mathrm{ppm}\right)$ & Elemental Analysis (CHN) \\
\hline 4 a & $\mathrm{H}-$ & $\begin{array}{l}\lambda_{\max } 290 \\
(\varepsilon 14523)\end{array}$ & $\begin{array}{l}1679.0(\text { coumarin, } C=0) \\
1634.74(C=N), 1491.00 \\
(C=C) .\end{array}$ & $\begin{array}{l}8.13(\mathrm{~s}, 1 \mathrm{H}, 4-\mathrm{H} \text { of coumarin) } 7.35-8.11 \\
(\mathrm{m}, 14 \mathrm{H}, \mathrm{Ar}-\mathrm{H}), 7.16 \\
(\mathrm{~s}, 1 \mathrm{H}, 4-\mathrm{H} \text { of pyrazole) }\end{array}$ & $\begin{array}{l}\text { Anal. Calcd for } \mathrm{C}_{24} \mathrm{H}_{16} \mathrm{~N}_{2} \mathrm{O}_{2} \\
\text { C, 79.11; } \mathrm{H}, 4.43 ; \mathrm{N}, 7.69 \text {. } \\
\text { Found: C, 79.14; } \mathrm{H}, 4.45 ; \mathrm{N}, 7.64 \text {. }\end{array}$ \\
\hline $4 \mathrm{~b}$ & 4-OMe- & $\begin{array}{l}\lambda_{\max } 272 \\
(\varepsilon 18673)\end{array}$ & $\begin{array}{l}1681.97 \text { (coumarin, } \\
C=0), 1624.74(C=N) \\
1490.05(C=C)\end{array}$ & $\begin{array}{l}8.13(\mathrm{~s}, 1 \mathrm{H}, 4-\mathrm{H} \text { of coumarin), } 7.01-7.88 \\
(\mathrm{m}, 13 \mathrm{H}, \mathrm{Ar}-\mathrm{H}), 7.15(\mathrm{~s}, 1 \mathrm{H}, 4-\mathrm{H} \text { of } \\
\text { pyrazole), } 3.81\left(\mathrm{~s}, 3 \mathrm{H},-\mathrm{OCH}_{3}\right) .\end{array}$ & $\begin{array}{l}\text { Anal. Calcd for } \mathrm{C}_{25} \mathrm{H}_{18} \mathrm{~N}_{2} \mathrm{O}_{3} \\
\text { C, 76.13; } \mathrm{H}, 4.60 ; \mathrm{N}, 7.10 \text {. } \\
\text { Found: } \mathrm{C}, 76.14 ; \mathrm{H}, 4.65 ; \mathrm{N}, 7.07 \text {. }\end{array}$ \\
\hline $4 c$ & $4-\mathrm{Cl}-$ & $\begin{array}{l}\lambda_{\max } 302 \\
(\varepsilon 12657)\end{array}$ & $\begin{array}{l}1682.05 \text { (coumarin, } \\
C=0), 1614.43(C=N), \\
1486.72(C=C) .\end{array}$ & $\begin{array}{l}8.15(\mathrm{~s}, 1 \mathrm{H}, 4-\mathrm{H} \text { of coumarin), } 7.31-8.13 \\
(\mathrm{m}, 13 \mathrm{H}, \mathrm{Ar}-\mathrm{H}), 7.27(\mathrm{~s}, 1 \mathrm{H}, 4-\mathrm{H} \text { of } \\
\text { pyrazole). }\end{array}$ & $\begin{array}{l}\text { Anal. Calcd for } \mathrm{C}_{24} \mathrm{H}_{15} \mathrm{ClN}_{2} \mathrm{O}_{2} \\
\text { C, } 72.27 ; \mathrm{H}, 3.79 ; \mathrm{N}, 7.02 \text {. } \\
\text { Found: } \mathrm{C}, 72.34 ; \mathrm{H}, 3.81 ; \mathrm{N}, 6.98 \text {. }\end{array}$ \\
\hline $4 d$ & $2,4-(\mathrm{Cl})_{2^{-}}$ & $\begin{array}{l}\lambda_{\max } 286 \\
(\varepsilon 16795)\end{array}$ & $\begin{array}{l}1682.63 \text { (coumarin, } \\
C=0), 1616.31(C=N), \\
1484.97(C=C) .\end{array}$ & $\begin{array}{l}8.15(\mathrm{~s}, 1 \mathrm{H}, 4-\mathrm{H} \text { of coumarin), } 7.31-8.08 \\
(\mathrm{m}, 12 \mathrm{H}, \mathrm{Ar}-\mathrm{H}), 7.28 \\
(\mathrm{~s}, 1 \mathrm{H}, 4-\mathrm{H} \text { of pyrazole) }\end{array}$ & $\begin{array}{l}\text { Anal. Calcd for } \mathrm{C}_{24} \mathrm{H}_{14} \mathrm{Cl}_{2} \mathrm{~N}_{2} \mathrm{O}_{2} \\
\text { C, } 66.53 .27 ; \mathrm{H}, 3.26 ; \mathrm{N}, 6.47 \text {. } \\
\text { Found: } \mathrm{C}, 66.56 ; \mathrm{H}, 3.29 ; \mathrm{N}, 6.41 \text {. }\end{array}$ \\
\hline $4 \mathrm{e}$ & 4-NMe $2^{-}$ & $\begin{array}{l}\lambda_{\max } 276 \\
(\varepsilon 17987)\end{array}$ & $\begin{array}{l}1673.65 \text { (coumarin, } \\
C=0), 1604.94(C=N), \\
1449.36(C=C) .\end{array}$ & $\begin{array}{l}8.13(\mathrm{~s}, 1 \mathrm{H}, 4-\mathrm{H} \text { of coumarin }), 6.77-7.88 \\
(\mathrm{~m}, 13 \mathrm{H}, \mathrm{Ar}-\mathrm{H}), 7.19(\mathrm{~s}, 1 \mathrm{H}, 4-\mathrm{H} \text { of } \\
\text { pyrazole), } 3.02\left(\mathrm{~s}, 6 \mathrm{H},-\mathrm{N}\left(\mathrm{CH}_{3}\right)_{2}\right) .\end{array}$ & $\begin{array}{l}\text { Anal. Calcd for } \mathrm{C}_{26} \mathrm{H}_{21} \mathrm{~N}_{3} \mathrm{O}_{2} \\
\text { C, 76.64; } \mathrm{H}, 5.19 ; \mathrm{N}, 10.31 \text {. } \\
\text { Found: } \mathrm{C}, 76.67 ; \mathrm{H}, 5.21 ; \mathrm{N}, 10.27 \text {. }\end{array}$ \\
\hline $4 f$ & $3-\mathrm{NO}_{2}^{-}$ & $\begin{array}{l}\lambda_{\max } 286 \\
(\varepsilon 15672)\end{array}$ & $\begin{array}{l}1685.23 \text { (coumarin, } \\
C=O), 1611.11(C=N), \\
1449.36(C=C) .\end{array}$ & $\begin{array}{l}8.69(\mathrm{~s}, 1 \mathrm{H}, 4-\mathrm{H} \text { of coumarin), } 7.16-7.88 \\
(\mathrm{m}, 13 \mathrm{H}, \mathrm{Ar}-\mathrm{H}), 7.14 \\
(\mathrm{~s}, 1 \mathrm{H}, 4-\mathrm{H} \text { of pyrazole). }\end{array}$ & $\begin{array}{l}\text { Anal. Calcd for } \mathrm{C}_{24} \mathrm{H}_{15} \mathrm{~N}_{3} \mathrm{O}_{4} \\
\text { C, } 70.41 ; \mathrm{H}, 3.69 ; \mathrm{N}, 10.26 \text {. } \\
\text { Found: } \mathrm{C}, 70.44 ; \mathrm{H}, 3.65 ; \mathrm{N}, 10.28 \text {. }\end{array}$ \\
\hline $4 \mathrm{~g}$ & 4-Me- & $\begin{array}{l}\lambda_{\max } 282 \\
(\varepsilon 17898)\end{array}$ & $\begin{array}{l}1715.41 \text { (coumarin, } \\
C=0), 1672.78(C=N), \\
1552.98(C=C) .\end{array}$ & $\begin{array}{l}8.13(\mathrm{~s}, 1 \mathrm{H}, 4-\mathrm{H} \text { of coumarin), } 7.12-7.88 \\
(\mathrm{m}, 13 \mathrm{H}, \mathrm{Ar}-\mathrm{H}), 7.23 \\
\left(\mathrm{~s}, 1 \mathrm{H}, 4-\mathrm{H} \text { of pyrazole), } 2.34\left(\mathrm{~s}, 3 \mathrm{H},-\mathrm{CH}_{3}\right) .\right.\end{array}$ & $\begin{array}{l}\text { Anal. Calcd for } \mathrm{C}_{25} \mathrm{H}_{18} \mathrm{~N}_{2} \mathrm{O}_{2} \\
\text { C, 79.35; } \mathrm{H}, 4.79 ; \mathrm{N}, 7.40 \text {. } \\
\text { Found: } \mathrm{C}, 79.38 ; \mathrm{H}, 4.80 ; \mathrm{N}, 7.36 \text {. }\end{array}$ \\
\hline $4 \mathrm{~h}$ & 3-OMe- & $\begin{array}{l}\lambda_{\max } 292 \\
(\varepsilon 12893)\end{array}$ & $\begin{array}{l}1681.97 \text { (coumarin, } \\
C=O), 1624.94(C=N), \\
1490.05(C=C) .\end{array}$ & $\begin{array}{l}8.13(\mathrm{~s}, 1 \mathrm{H}, 4-\mathrm{H} \text { of coumarin), } 7.02-7.88 \\
(\mathrm{m}, 13 \mathrm{H}, \mathrm{Ar}-\mathrm{H}), 7.26(\mathrm{~s}, 1 \mathrm{H}, 4-\mathrm{H} \text { of } \\
\text { pyrazole), } 3.81\left(\mathrm{~s}, 3 \mathrm{H},-\mathrm{OCH}_{3}\right)\end{array}$ & $\begin{array}{l}\text { Anal. Calcd for } \mathrm{C}_{25} \mathrm{H}_{18} \mathrm{~N}_{2} \mathrm{O}_{3} \\
\text { C, 76.13; } \mathrm{H}, 4.60 ; \mathrm{N}, 7.10 \text {. } \\
\text { Found: } \mathrm{C}, 76.14 ; \mathrm{H}, 4.65 ; \mathrm{N}, 7.07 \text {. }\end{array}$ \\
\hline $4 i$ & $4-\mathrm{F}-$ & $\begin{array}{l}\lambda_{\max } 312 \\
(\varepsilon 12331)\end{array}$ & $\begin{array}{l}1682.05 \text { (coumarin, } \\
C=O), 1614.43(C=N), \\
1486.72(C=C) .\end{array}$ & $\begin{array}{l}8.13(\mathrm{~s}, 1 \mathrm{H}, 4-\mathrm{H} \text { of coumarin), } 7.28-7.88 \\
(\mathrm{m}, 13 \mathrm{H}, \mathrm{Ar}-\mathrm{H}), 7.16(\mathrm{~s}, 1 \mathrm{H}, 4-\mathrm{H} \text { of } \\
\text { pyrazole). }\end{array}$ & $\begin{array}{l}\text { Anal. Calcd for } \mathrm{C}_{24} \mathrm{H}_{15} \mathrm{FN}_{2} \mathrm{O}_{2} \\
\text { C, 75.38; } \mathrm{H}, 3.95 ; \mathrm{N}, 7.33 \text {. } \\
\text { Found: } \mathrm{C}, 75.41 ; \mathrm{H}, 3.97 ; \mathrm{N}, 7.30 \text {. }\end{array}$ \\
\hline $4 j$ & $2-\mathrm{NO}_{2}^{-}$ & $\begin{array}{l}\lambda_{\max } 276 \\
(\varepsilon 13892)\end{array}$ & $\begin{array}{l}1689.61 \text { (coumarin, } \\
C=O), 1609.40(C=N) \\
1458.55(C=C)\end{array}$ & $\begin{array}{l}8.16(\mathrm{~s}, 1 \mathrm{H}, 4-\mathrm{H} \text { of coumarin), } 7.37-8.13 \\
(\mathrm{m}, 13 \mathrm{H}, \mathrm{Ar}-\mathrm{H}), 6.99(\mathrm{~s}, 1 \mathrm{H}, 4-\mathrm{H} \text { of } \\
\text { pyrazole). }\end{array}$ & $\begin{array}{l}\text { Anal. Calcd for } \mathrm{C}_{24} \mathrm{H}_{15} \mathrm{~N}_{3} \mathrm{O}_{4} \\
\mathrm{C}, 70.41 ; \mathrm{H}, 3.69 ; \mathrm{N}, 10.26 \text {. } \\
\text { Found: } \mathrm{C}, 70.44 ; \mathrm{H}, 3.65 ; \mathrm{N}, 10.28 \text {. }\end{array}$ \\
\hline $4 k$ & $4-\mathrm{OH}-$ & $\begin{array}{l}\lambda_{\max } 274 \\
(\varepsilon 12891)\end{array}$ & $\begin{array}{l}3208.00(-\mathrm{OH}), 1671.33 \\
\text { (coumarin, } \mathrm{C}=\mathrm{O}) \text {, } \\
1607.79,1505.92(\mathrm{C}=\mathrm{C})\end{array}$ & $\begin{array}{l}9.46(\mathrm{~s}, 1 \mathrm{H},-\mathrm{OH}), 8.13(\mathrm{~s}, 1 \mathrm{H}, 4-\mathrm{H} \text { of } \\
\text { coumarin), 6.85-7.66 (m, 13H, Ar-H), } 7.15 \\
\text { (s, } 1 \mathrm{H}, 4-\mathrm{H} \text { of pyrazole) }\end{array}$ & $\begin{array}{l}\text { Anal. Calcd for } \mathrm{C}_{24} \mathrm{H}_{16} \mathrm{~N}_{2} \mathrm{O}_{3} \\
\text { C, 75.78; } \mathrm{H}, 4.24 ; \mathrm{N}, 7.36 \text {. } \\
\text { Found: } \mathrm{C}, 75.81 ; \mathrm{H}, 4.26 ; \mathrm{N}, 7.33 \text {. }\end{array}$ \\
\hline
\end{tabular}

body weight and degree of secondary lesions were recorded daily from day 16 to 19 . The decrease or increase, in mean paw volume per group per day was calculated as a percent inhibition from day 15 onwards.

\section{Ulcerogenic activity [27]}

Albino rats of either sex were divided into control, standard and different test groups of 6 animals each group (170-250g). They were starved for $48 \mathrm{~h}$ (water ad libitum) prior to drug administration. Control group received only $0.5 \%$ sodium CMC solution, standard group was orally administered with acetylsalicylic acid in sodium CMC solution and test compounds $\mathbf{4 c}, \mathbf{d}, \mathbf{h}$ and $\mathbf{i}$ were administered orally at the dose of $200 \mathrm{mg} / \mathrm{kg}$ b.w. All animals were sacrificed after $7 \mathrm{~h}$ of drug administration. Stomach was removed and placed on saline-soaked filter paper until inspection. A longitudinal incision along the greater curvature was made with fine scissors. The stomach was everted over the index finger and the presence or absence of gastric irritationwas determined. The ulcer index for each group was determined according to a previously reported method [36] by counting the number of lesions $(x)$ in each of 5 size classes $(y)$. The classes were defined as $=1$ (pinpoint lesion), $y=2$ (lesions $<1 \mathrm{~mm}$ diameter), $\mathrm{y}=3$ (lesions $1-2 \mathrm{~mm}$ diameter), $\mathrm{y}=4$ (lesions $2-4 \mathrm{~mm}$ diameter) and $\mathrm{y}=5$ (lesions $>4 \mathrm{~mm}$ diameter). The ulcer index was calculated using $\sum 5_{\mathrm{i}=1} \mathrm{X}_{\mathrm{i}} \mathrm{y}_{\mathrm{i}}$

\section{Antioxidant Activity}

Free radical scavenging activity of compounds was determined using 1,1-diphenyl-2-picryl hydrazyl (DPPH) free radical [28].
Briefly, $2 \mathrm{~mL}$ samples of various concentrations (100-1000 $\mu \mathrm{g}$ / $\mathrm{ml}$ ) were added to $2 \mathrm{~mL}$ of $100 \mu \mathrm{M}$ DPPH solution. After $20 \mathrm{~min}$ incubation at room temperature, the absorbance was read against a blank at $517 \mathrm{~nm}$. The change in absorbance with respect to the control (containing DPPH only without sample, expressed as $100 \%$ free radicals) was calculated as percentage scavenging using following the equation:

$\left(A_{517}\right.$ blank $-A_{517}$ sample $) \div A_{517}$ blank $\times 100 \%$.

The reading was taken in triplicate and mean used for calculation of $\mathrm{IC}_{50}$. The $\mathrm{IC}_{50}$ (mean $\pm \mathrm{SEM}$ ) stand for the concentration required for $50 \%$ inhibition of DPPH radicals and was calculated from ORIGIN PC version 6.0 software.

\section{Statistical analysis}

Data are presented as arithmetic mean \pm SEM. Statistical analysis was performed by one way variance (ANOVA) followed by Dunnett's test,"p" value of less than 0.05 was considered as statistically significant using GRAPHPAD PRISM PC version 4.03 by GraphPad software INC-2005.

\section{Results and Discussion}

Synthesis

The synthesis of 3-[3-(substituted phenyl)-1-phenyl-1H-pyrazol-5-yl]-2H-chromen-2-one (4a-k) was carried out as pre- 
Table 3 In vivo acute anti-inflammatory activity of 3-[3-(substituted phenyl)-1-phenyl-1H-pyrazol-5-yl]-2H-chromen-2-one derivatives (4a-k) in carrageenaninduced paw edema.

\begin{tabular}{|c|c|c|c|c|c|c|c|}
\hline \multirow[t]{2}{*}{ Compound } & \multirow[t]{2}{*}{$\mathbf{R}$} & \multicolumn{6}{|c|}{ Paw edema volume in $\mathrm{ml}$ (\% inhibition) } \\
\hline & & $\mathbf{O h}$ & $1 \mathrm{~h}$ & $2 \mathrm{~h}$ & $3 \mathrm{~h}$ & $4 \mathrm{~h}$ & $5 \mathrm{~h}$ \\
\hline Control & & $0.50 \pm 0.026$ & $0.89 \pm 0.02$ & $1.11 \pm 0.030$ & $1.18 \pm 0.012$ & $1.26 \pm 0.008$ & $1.22 \pm 0.007$ \\
\hline $4 a$ & $\mathrm{H}-$ & $0.47 \pm 0.020$ & $\begin{array}{l}0.81 \pm 0.019 \\
(8.86)\end{array}$ & $\begin{array}{l}0.92 \pm 0.011 \\
(16.28)^{* *}\end{array}$ & $\begin{array}{l}0.84 \pm 0.019 \\
(28.68)^{* *}\end{array}$ & $\begin{array}{l}0.87 \pm 0.021 \\
(30.79)^{* *}\end{array}$ & $\begin{array}{l}0.81 \pm 0.021 \\
(33.01)^{* *}\end{array}$ \\
\hline $4 b$ & 4-OMe- & $0.46 \pm 0.009$ & $\begin{array}{l}0.78 \pm 0.017 \\
(12.44)^{* *}\end{array}$ & $\begin{array}{l}0.90 \pm 0.014 \\
(18.82)^{* *}\end{array}$ & $\begin{array}{l}0.94 \pm 0.012 \\
(20.31)^{* *}\end{array}$ & $\begin{array}{l}0.88 \pm 0.021 \\
(29.77)^{* *}\end{array}$ & $\begin{array}{l}0.81 \pm 0.019 \\
(33.42)^{* *}\end{array}$ \\
\hline $4 c$ & $4-\mathrm{Cl}$ & $0.45 \pm 0.021$ & $\begin{array}{l}0.76 \pm 0.019 \\
(14.31)^{* *}\end{array}$ & $\begin{array}{l}0.79 \pm 0.019 \\
(28.26)^{* *}\end{array}$ & $\begin{array}{l}0.76 \pm 0.015 \\
(35.11)^{* *}\end{array}$ & $\begin{array}{l}0.64 \pm 0.019 \\
(48.94)^{* *}\end{array}$ & $\begin{array}{l}0.55 \pm 0.017 \\
(54.57)^{* *}\end{array}$ \\
\hline $4 d$ & $2,4-(\mathrm{Cl})_{2}$ & $0.48 \pm 0.018$ & $\begin{array}{l}0.80 \pm 0.030 \\
(9.26)\end{array}$ & $\begin{array}{l}0.74 \pm 0.019 \\
(33.31)^{* *}\end{array}$ & $\begin{array}{l}0.69 \pm 0.024 \\
(41.60)^{* *}\end{array}$ & $\begin{array}{l}0.64 \pm 0.024 \\
(49.19)^{* *}\end{array}$ & $\begin{array}{l}0.61 \pm 0.023 \\
(50.06)^{* *}\end{array}$ \\
\hline $4 \mathrm{e}$ & 4-NMe ${ }_{2}$ & $0.49 \pm 0.04$ & $\begin{array}{l}0.79 \pm 0.010 \\
(10.88)^{*}\end{array}$ & $\begin{array}{l}0.78 \pm 0.014 \\
(28.99)^{* *}\end{array}$ & $\begin{array}{l}0.71 \pm 0.013 \\
(39.13)^{* *}\end{array}$ & $\begin{array}{l}0.66 \pm 0.017 \\
(47.42)^{* *}\end{array}$ & $\begin{array}{l}0.61 \pm 0.017 \\
(49.37)^{* *}\end{array}$ \\
\hline $4 f$ & $3-\mathrm{NO}_{2}$ & $0.48 \pm 0.016$ & $\begin{array}{l}0.82 \pm 0.029 \\
(7.91)\end{array}$ & $\begin{array}{l}0.88 \pm 0.021 \\
(20.60)^{* *}\end{array}$ & $\begin{array}{l}0.84 \pm 0.019 \\
(28.20)^{* *}\end{array}$ & $\begin{array}{l}0.82 \pm 0.020 \\
(34.69)^{* *}\end{array}$ & $\begin{array}{l}0.78 \pm 0.019 \\
(35.74)^{* *}\end{array}$ \\
\hline $4 g$ & 4-Me & $0.47 \pm 0.019$ & $\begin{array}{l}0.80 \pm 0.022 \\
(9.63)\end{array}$ & $\begin{array}{l}0.93 \pm 0.011 \\
(15.95)^{* *}\end{array}$ & $\begin{array}{l}0.85 \pm 0.017 \\
(27.64)^{* *}\end{array}$ & $\begin{array}{l}0.83 \pm 0.020 \\
(34.03)^{* *}\end{array}$ & $\begin{array}{l}0.82 \pm 0.024 \\
(32.87)^{* *}\end{array}$ \\
\hline $4 \mathrm{~h}$ & 3-OMe & $0.46 \pm 0.017$ & $\begin{array}{l}0.81 \pm 0.017 \\
(8.47)\end{array}$ & $\begin{array}{l}0.76 \pm 0.017 \\
(31.11)^{* *}\end{array}$ & $\begin{array}{l}0.71 \pm 0.018 \\
(39.63)^{* *}\end{array}$ & $\begin{array}{l}0.63 \pm 0.013 \\
(49.36)^{* *}\end{array}$ & $\begin{array}{l}0.54 \pm 0.026 \\
(55.25)^{* *}\end{array}$ \\
\hline $4 \mathrm{i}$ & $4-\mathrm{F}$ & $0.44 \pm 0.013$ & $\begin{array}{l}0.75 \pm 0.017 \\
(15.24)^{* *}\end{array}$ & $\begin{array}{l}0.79 \pm 0.010 \\
(28.26)^{* *}\end{array}$ & $\begin{array}{l}0.75 \pm 0.014 \\
(35.82)^{* *}\end{array}$ & $\begin{array}{l}0.63 \pm 0.016 \\
(49.73)^{* *}\end{array}$ & $\begin{array}{l}0.50 \pm 0.013 \\
(58.52)^{* *}\end{array}$ \\
\hline $4 \mathrm{j}$ & $2-\mathrm{NO}_{2}$ & $0.49 \pm 0.019$ & $\begin{array}{l}0.79 \pm 0.023 \\
(10.75)^{*}\end{array}$ & $\begin{array}{l}0.92 \pm 0.014 \\
(16.85)^{* *}\end{array}$ & $\begin{array}{l}0.88 \pm 0.014 \\
(25.24)^{* *}\end{array}$ & $\begin{array}{l}0.84 \pm 0.019 \\
(32.98)^{* *}\end{array}$ & $\begin{array}{l}0.83 \pm 0.019 \\
(31.51)^{* *}\end{array}$ \\
\hline $4 k$ & $4-\mathrm{OH}$ & $0.48 \pm 0.015$ & $\begin{array}{l}0.76 \pm 0.025 \\
(13.75)^{* *}\end{array}$ & $\begin{array}{l}0.88 \pm 0.021 \\
(20.75)^{* *}\end{array}$ & $\begin{array}{l}0.80 \pm 0.025 \\
(32.29)^{* *}\end{array}$ & $\begin{array}{l}0.74 \pm \pm 0.026 \\
(41.29)^{* *}\end{array}$ & $\begin{array}{l}0.67 \pm 0.024 \\
(44.61)^{* *}\end{array}$ \\
\hline Diclofenac & & $0.50 \pm 0.022$ & $\begin{array}{l}0.76 \pm 0.014 \\
(14.01)^{* * *}\end{array}$ & $\begin{array}{l}0.79 \pm 0.017 \\
(28.81)^{* * *}\end{array}$ & $\begin{array}{l}0.59 \pm 0.018 \\
(49.60)^{* * *}\end{array}$ & $\begin{array}{l}0.46 \pm 0.020 \\
(63.19)^{* * *}\end{array}$ & $\begin{array}{l}0.41 \pm 0.013 \\
(66.43)^{* * *}\end{array}$ \\
\hline
\end{tabular}

The results are expressed as mean \pm SEM $(n=6)$. Each value in parenthesis indicates the percentage inhibition. Significance was calculated by using one-way ANOVA. The difference in results was considered significant when $\mathrm{p}<0.05$. ${ }^{*} \mathrm{p}<0.05 \mathrm{vs}$. control at $200 \mathrm{mg} / \mathrm{kg}$ b.w; ${ }^{* *} \mathrm{p}<0.01 \mathrm{vs}$. control at $200 \mathrm{mg} / \mathrm{kg}$ b.w; ${ }^{* * *} \mathrm{p}<0.01 \mathrm{vs}$. control at $13.5 \mathrm{mg} / \mathrm{kg}$ b.w

Table 4 In vivo chronic anti-inflammatory activity of selected compounds in adjuvant induced arthritis model.

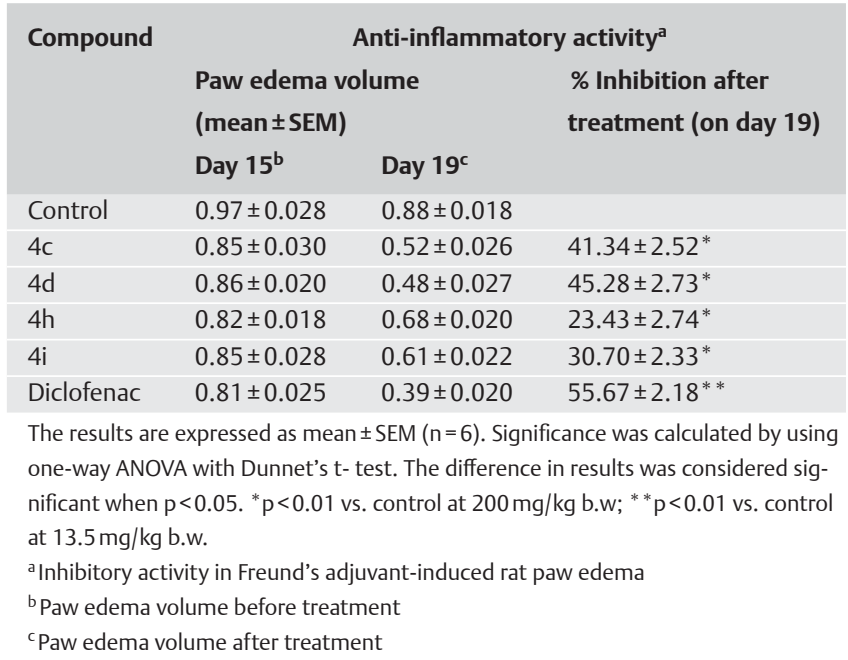

sented in $\odot$ Fig. 1. Starting material 3-acetyl-2H-chromen-2-one 1 was synthesized by the reaction of salicylaldehyde with ethylacetoacetate in the presence of a catalytic amount of piperidine at room temperature following the literature procedure [22]. 3-[(2E)-3-(substitutedphenyl)prop-2-enoyl]-2H-chromen-2one (chalcones, 2a-k) were synthesized by Claisen-Schmidt condensation of 3-acetyl-2H-chromen- 2-one $\mathbf{1}$ with various substituted benzaldehydes in the presence of a mixture of piperidine and n-butanol. Efforts to convert compounds $\mathbf{2 a - k}$ into target molecules $\mathbf{4 a - k}$ under a variety of conditions were not successful. Hence, an alternative method was adopted. This involved the bromination of chalcones $\mathbf{2 a - k}$ and subsequent ring closure using phenyl hydrazine. Bromination of chalcones $\mathbf{2 a - k}$ was carried out in chloroform using bromine in chloroform to yield dibromo compounds 3a-k, which were cyclized with phenyl hydrazine in the presence of triethylamine in absolute ethanol to afford the 3-[3-(substituted phenyl)-1-phenyl-1Hpyrazol-5-yl]-2H-chromen-2-one 4a-k. The physicochemical and spectral analysis data of the synthesized compounds are depicted in $\odot$ Table 1, 2, respectively.

\section{Acute toxicity study}

From the preliminary toxicity studies, it was observed that, all the test compounds have revealed good safety profile till the uppermost dose $(2000 \mathrm{mg} / \mathrm{kg})$. No mortality of animals observed even after $24 \mathrm{~h}$ but there were few changes in the behavioral response like alertness, touch response and restlessness. Therefore, $1 / 10^{\text {th }}$ of the maximum tolerated dose i.e., $200 \mathrm{mg} / \mathrm{kg}$ b.w. was chosen for the various pharmacological evaluations.

\section{Acute anti-inflammatory activity}

- Table 3 reveals the in vivo acute anti-inflammatory activity of a novel series of 3-[3-(substituted phenyl)-1-phenyl-1H-pyrazol-5-yl]-2H-chromen-2-one (4a-k) at a dose of $200 \mathrm{mg} / \mathrm{kg}$ in carrageenan-induced paw edema method. Carrageenan-induced edema is a non-specific inflammation resulting from a complex of diverse mediators. Since edema of this type is highly sensitive to NSAIDs, carrageenan has been accepted as a useful agent for studying new anti-inflammatory agents. This model reliably predicts the anti-inflammatory efficacy of the NSAIDs, and during the second phase it detects compounds that are anti-inflammatory agents as a result of inhibition of prostaglandin amplification [35]. As shown in $\odot$ Table 3, the entire investigated 


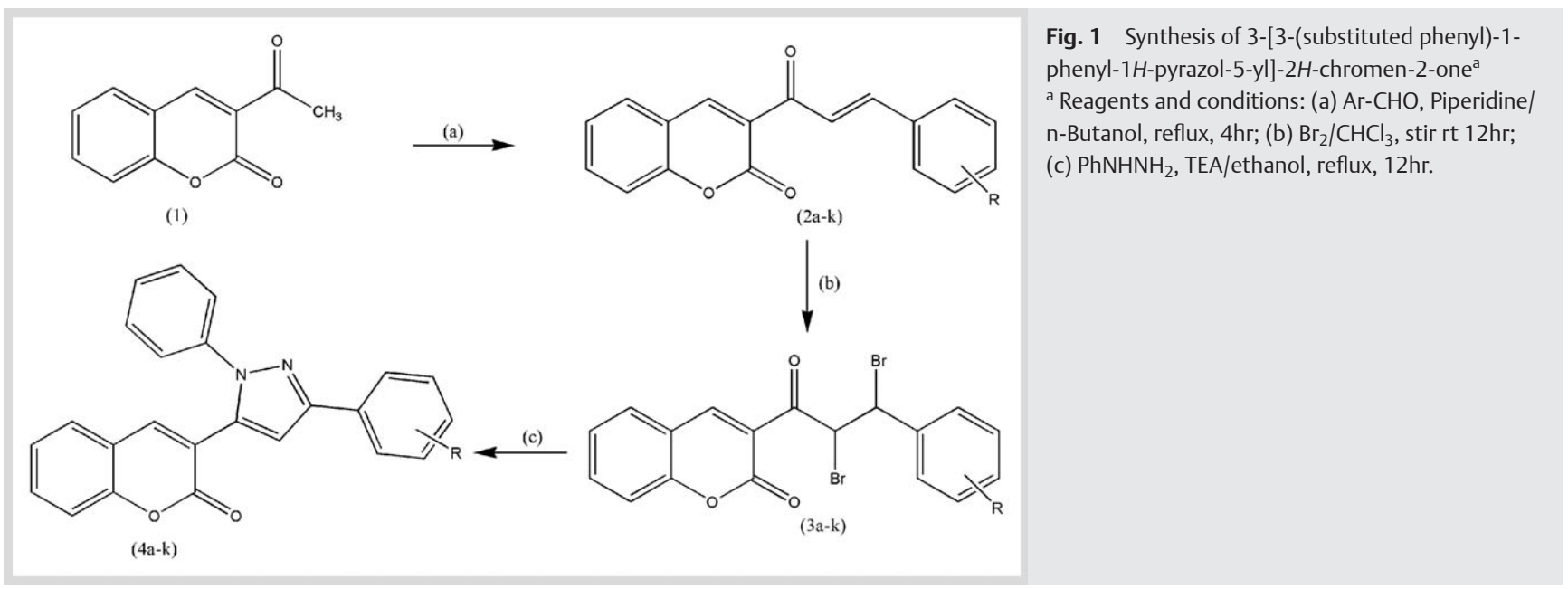

compounds exhibited moderate to good anti-inflammatory activity with the percentage inhibition of edema formation ranged from 31.51 to 66.43 , while the reference drug diclofenac $(13.5 \mathrm{mg} / \mathrm{kg}$ ) showed $66.43 \%$ inhibition at fifth hour. Compound 4c (35.1 and 54.57\%) and 4d (41.60 and 50.06\%) showed good inhibitory activity at third and fifth hour respectively, while the most active compounds $\mathbf{4 i}$ (35.82 and 58.52\%) and $\mathbf{4 h}$ (39.63 and $55.25 \%$ ) among the series, exhibited the excellent inhibitory activity at third and fifth hour, respectively. The anti-inflammatory activity of these compounds was comparable with that of the standard drug diclofenac, although not equal. Compounds that showed significant anti-inflammatory activity profile were further tested for chronic anti-inflammatory and ulcerogenic activities.

\section{Chronic anti-inflammatory activity}

- Table 4 explains the in vivo chronic anti-inflammatory activity of compounds $\mathbf{4 c}, \mathbf{d}, \mathbf{h}$ and $\mathbf{i}$ in adjuvant-induced arthritis in rats. Injection of Freund's complete adjuvant to the hind footpad in rats produced arthritis and rats with developed arthritis were chosen for the study. On day 15 , there were not many variations in the edema volumes of different groups. Administration of $\mathbf{4 c}$, $\mathbf{d}, \mathbf{h}$ and $\mathbf{i}$ at a dose of $200 \mathrm{mg} / \mathrm{kg}$ and diclofenac $(13.5 \mathrm{mg} / \mathrm{kg}$ ) for 3 days (from day 16 to day 19) in rats has produced significant reduction in paw volume (by day 19) when compared to the arthritic control group. Compounds 4c (41.32\%), 4d (45.28\%) and $4 \mathbf{i}(30.75 \%)$ were found to possess a substantial inhibition of edema formation when compared to $\mathbf{4 h}(23.58 \%)$ while diclofenac (55.66\%) exhibited significant anti-inflammatory activity.

\section{Ulcerogenic activity}

The major drawback of NSAIDs is their gastric ulcer formation due to gastric irritation. The extent of ulcerogenic effect was evaluated for compounds $\mathbf{4 c}, \mathbf{d}, \mathbf{h}$ and $\mathbf{i}$ in rat stress model at the therapeutic dose (i.e., $200 \mathrm{mg} / \mathrm{kg}$ b.w.). The gastric ulcerogenic potential was evaluated by calculating the ulcer index in treated and control animals. Results are given in 0 Table 5 that indicates these four compounds (ulcer index ranges from 1.8 to 4.6) cause less gastric ulceration and disruption of gastric epithelial cells at the abovementioned oral dose as compared to acetylsalicylic acid (ulcer index 5.1). Hence gastric tolerance to these compounds was better than that of standard drug.
Table 5 Ulcerogenic activity of selected compounds in comparison with acetylsalicylic acid.

\begin{tabular}{|lc|}
\hline Compound & Ulcer Index $( \pm$ SEM) \\
\hline Control & $1.4( \pm 3.10)$ \\
\hline $4 \mathrm{c}$ & $1.8( \pm 0.42)$ \\
\hline $4 \mathrm{~d}$ & $2.67( \pm 0.28)^{*}$ \\
\hline $4 \mathrm{~h}$ & $2.12( \pm 0.11)$ \\
\hline $4 \mathrm{i}$ & $4.6( \pm 1.02)$ \\
\hline Acetylsalicylic acid & $5.1( \pm 0.88)^{* *}$ \\
\hline
\end{tabular}

The results are expressed as mean \pm SEM $(n=6)$. Data analysed by one-way ANOVA with Dunnet's t-test. ${ }^{*} \mathrm{p}<0.05$ significant from control; ${ }^{* *} \mathrm{p}<0.01$ significant from control

Table 6 Antioxidant activity of 3-[3-(substituted phenyl)-1-phenyl-1Hpyrazol-5-yl]-2H-chromen-2-one derivatives (4a-k).

\begin{tabular}{|c|c|}
\hline Compound & $\begin{array}{l}\text { DPPH scavenging activity } I_{50} \\
\left( \pm \text { SEM) }(\mathrm{mM})^{\mathrm{a}}\right.\end{array}$ \\
\hline $4 \mathrm{a}$ & $1.77 \pm 0.012$ \\
\hline $4 \mathrm{~b}$ & $1.51 \pm 0.012$ \\
\hline $4 c$ & $1.27 \pm 0.133$ \\
\hline $4 d$ & $1.03 \pm 0.067$ \\
\hline $4 \mathrm{e}$ & $1.23 \pm 0.016$ \\
\hline $4 \mathrm{f}$ & $1.15 \pm 0.045$ \\
\hline $4 \mathrm{~g}$ & $1.37 \pm 0.167$ \\
\hline $4 \mathrm{~h}$ & $1.59 \pm 0.023$ \\
\hline $4 i$ & $1.32 \pm 0.059$ \\
\hline $4 \mathrm{j}$ & $1.52 \pm 0.025$ \\
\hline $4 k$ & $1.07 \pm 0.080$ \\
\hline Butylated Hydroxy Toluene. & $0.13 \pm 0.012$ \\
\hline Ascorbic Acid & $0.026 \pm 0.022$ \\
\hline \multicolumn{2}{|c|}{$\begin{array}{l}\text { a The results are expressed as } \mathrm{IC}_{50} \pm \mathrm{SEM}(\mathrm{n}=3)(\mathrm{mM}) \text {, the concentration of the test } \\
\text { compound that provides } 50 \% \text { scavenging of the DPPH radicals already available in } \\
\text { the solution. Butylated hydroxy toluene }(\mathrm{BHT}) \text { and Ascorbic acid }(\text { ASC) are used as } \\
\text { the standard reference }\end{array}$} \\
\hline
\end{tabular}

\section{Antioxidant activity}

It has been reported that scavenging of ROS might be beneficial in reducing the development of inflammation. In order to determine the extent of scavenging effect, all compounds were tested for antioxidant activity using 1,1-diphenyl-2-picryl hydrazyl (DPPH) free radical. All compounds showed antioxidant activity ( $\bullet$ Table 6 ). Compounds $4 \mathbf{d}$ and $\mathbf{k}$ showed $1.03 \pm 0.067$ and $1.07 \pm 0.088 \mathrm{IC}_{50}$ as compared with standard ascorbic acid $(0.026 \pm 0.022 \mathrm{mM})$ and butylated hydroxy toluene $(0.13 \pm 0.012 \mathrm{mM})$. 


\section{Conclusion}

\section{$\nabla$}

The objective of the present study was to synthesize and investigate the anti-inflammatory and antioxidant activities of a new series of pyrazolylcoumarins with the hope of discovering new structure leads serving as dual anti-inflammatory-antiantioxidant agents. The substitution pattern of the pyrazole ring was rationalized so as to be correlated to the vicinal diaryl heterocycles template. Amongst the tested compounds, $\mathbf{4 h}$ and $\mathbf{4 i}$ showed pronounced acute antiinflammatory activity ( 55 and $58 \%$ respectively) that was comparable to diclofenac (66\%), whereas, compounds $\mathbf{4 c}$ and $\mathbf{4 d}$ displayed good anti-inflammatory activity (54 and 50\% respectively) while compound 4d showed considerable activity in model of chronic inflammation such as adjuvant-induced arthritis.. However, none of the newly synthesized compounds was found to be superior over the reference drug. In addition, the tested compounds were also found to possess less degree of ulcerogenic potential as compared to aspirin. Compounds $\mathbf{4 d}$ and $\mathbf{4 k}$ having dichloro and hydroxyl moiety demonstrated maximum antioxidant activity.

The compound with p-chlorophenyl (4c) substitution was found to be active among tested compounds as anti-inflammatory as well as antioxidant agents, amd less degree of ulcerogenic potential. Here strong electron withdrawing $(-\mathrm{Cl})$ substitution at ortho and para position in phenyl ring responsible for activity. This shows that the antioxidant activity influence the antiinflammatory activity. Finally compound $\mathbf{4 c}$ could be identified as the most biologically active member within this study with an interesting dual anti-inflammatory and antioxidant profile. Further in-vitro COX-1 and 2 inhibitory activity of pyrazolylcoumarins class needs to be investigated using celecoxib as standard with the hope of finding new anti-inflammatory agent.

\section{Acknowledgement \\ $\nabla$}

The authors are thankful to Principal, RVS College of Pharmaceutical Science, Sulur, Coimbtore We are grateful to The Director, SAIF, Punjab University for providing spectral analysis.

\section{Conflict of Interest \\ $\nabla$}

Authors hereby declare that there are no financial/commercial conflicts of interest.

\section{References}

1 Sontag SJ. Prostaglandins in peptic ulcer disease: An overview of current status and future directions. Drug 1986; 32: 445-457

2 Allison MC, Howatson AG, Torrance CJ et al. Gastrointestinal damage associated with the use of nonsteroidal antiinflammatory drugs. N Engl J Med 1992; 327: 749-754

3 Flower RJ. The development of COX2 inhibitors. Nature Reviews Drug Discovery 2003; 2: 179-191

4 Clive DM, Stoff JS. Renal syndromes associated with non-steroidal antiinflammatory drugs. N Engl J Med 1984; 310: 563-570
5 Song Y, Connor DT, Doubleday R et al. Synthesis, structure activity relationship and in-vivo evaluation of substituted ditetrabutyl phenols as a novel class of potent selective and orally active cyclooxygenase- 2 inhibitors, 1-thiazolone and Oxazolone series. J Med Chem 1999; 42: $1151-1160$

6 Chandrasekharan NV, Dai H, Roos KLT et al. COX-3, a cyclooxygenase-1 variant inhibited by acetaminophen and other analgesic/antipyretic drugs: cloning, structure and expression. Proc Natl Acad Sci USA 2002; 99: 13926-13931

7 Palomer A, Cabré F, Pascual J et al. Identification of novel cyclooxygenase-2 selective inhibitors using pharmacophore models. J Med Chem 2002; 45 (7): 1402-1411

8 Gadad AK, Kittur BS, Kapsi SG et al. Synthesis, analgesic and antiinflammatory activities of some 1-acyl/aracyl-5-aminopyrazole derivatives. Arzneim Forsch 1996; 46: 1082-1085

9 Penning TD, Talley JJ et al. Synthesis and biological evaluation of the 1,5-diarylpyrazole class of cyclooxygenase-2 inhibitors: Identification of 4-5-(4-methylphenyl)-3-(trifluoromethyl)-1 H-pyrazol-1-yl]benzenesulfonamide (SC-58635, Celecoxib). J Med Chem 1997; 40: 1347-1365

10 Tsuji K, Nakamura K, Ogino $T$ et al. Studies on anti-inflammatory agents. VI. Synthesis and pharmacological properties of 2,3-diarylthiophenes. Chem Pharm Bull 1998; 46: 279-286

11 Connolly PJ, Wetter SK, Beers KN et al. N-hydroxyurea and hydroxamic acid inhibitors of cyclooxygenase and 5-lipoxygenase. Bioorg Med Chem Lett 1999; 9: 979-986

12 Boschelli D, Connor D, Bornemeier DA et al. 1,3,4-oxadiazole, 1, 3, 4-thiadiazole, and 1, 2, 4-triazole analogs of the fenamates: In vitro inhibition of cyclooxygenase and 5-lipoxygenase activities. J Med Chem 1993; 36: $1802-1810$

13 Freeman B, Crop J. Biology of disease, free radicals and tissue injury. Lab Invest 1982; 47: 412-426

14 Vane J. The mode of action of asprin-like drugs. Agents React 1978; 6: $430-431$

15 Halliwell B, Gutteridge JMC. Free radicals in biology and medicine. $2^{\text {nd }}$ ed. Oxford: Clarendon Press, 1989; 427

16 Roberfroid $M$, Viche H, Remede J. Free Radicals in Drug Research. In: Testa B (ed.). adv. Drug Research Res. Vol 16:Academic Press, London: $1987 ; 2-43$

17 Velavan $S$, Naghlendran $K$, Mahesh $R$ et al. In vitro antioxidant activity of Asparagus racemosus root. Phcogmag 1998; 26-32

18 Kennedy R, Thornes R. Coumarins: Biology, Applications and Mode of Action. Wiley \& Sons, Chichester, UK: 1997

19 Murray RDH, Mendez J, Brown SA. The Natural Coumarins, Occurrence, Chemistry and Biochemistry. John Wiely \& Sons, New York: 1982

20 Fylaktakidou KC, Hadjipavlou-Litina DJ, Litinas KE et al. Natural and Synthetic Coumarin Derivatives with Antiinflammatory/Antioxidant Activities. Curr Pharm Design 2004; 10: 3813-3833

21 Lacy A, O'Kennedy R. Studies on coumarins and coumarin-related compounds to determine their therapeutic role in the treatment of cancer. Curr Pharm Des 2004; 10: 3797-3811

22 Knoevenagel E. Knoevenagel Condensation. Chem Ber 1898; 31: 2596

23 Aragade P, Venkatnarayanan R, Maddi VS et al. J. Synthesis and antibacterial activity of a new series of 3-[3-(substituted phenyl)-1-phenyl-1H-pyrazol-5-yl]-2H-chromen-2-one derivative. Enzy Inhib Med Chem 2012; 27: 849-853

24 OECD/OCDC, OECD "Guidelines for Testing of Chemicals" Revised Draft Guidelines 423; Acute Oral Toxicity Class Method, Revised Document, October 2000

25 Winter CA, Risley EA, Nuss GW. Carrageenin-induced edema in hind paw of the rat as an assay for antiiflammatory drugs. Proc Soc Exp Biol Med 1962; 111: 544-547

26 Newbould BB. Chemotherapy of arthritis induced in rats by Mycobacterial adjuvant. $\mathrm{Br} \mathrm{j}$ pharmacol chemother 1963; 21: 127-136

27 Vogel HG (ed.). Drug Discovery and Evaluation: Pharmacological Assays. third ed. Springer-Verlag, Berlin, Heidelberg: 2002

28 Blois MS. Antioxidant determination by the use of a stable free radical. Nature 1958; 181: 1199-1200 